\title{
Application of Improved BP Neural Network Based on Genetic Algorithm in Fault Diagnosis of Equipment
}

\author{
Xin $\operatorname{Ren}^{1,2, a}$ \\ ${ }^{1}$ Graduate School, National Defense University, Beijing, 100091, China \\ ${ }^{2}$ Medical Protection Laboratory, Naval Medical Research Institute, Shanghai, 200433, China \\ aemail: renxins841013@163.com
}

Keywords: equipment; fault diagnosis; BP neural network; genetic algorithm

\begin{abstract}
Aiming at the problems of traditional BP neural network in fault diagnosis of equipment, the genetic algorithm is introduced to optimize the network, and the fault diagnosis model of equipment is established. The modeling ideas and considerations are introduced in detail, and the simulation calculation is carried out. The results show that the improved network has a good approximation performance, the training speed and accuracy are greatly improved, and it can be better to carry out fault diagnosis of equipment.
\end{abstract}

\section{Introduction}

Equipment is system very complicated, and it is very difficult to carry out the fault diagnosis of equipment. As a forward feedback network, BP neural network is a kind of the most mature network, which has been widely used in fault diagnosis. But because of some inherent defects, the application of network has been limited in fault diagnosis of equipment.

\section{Basic principles and improvement of BP neural network diagnosis}

2.1 Basic principles of BP neural network

BP network is proposed by a group of scientists headed by Rumelhart and McCelland in 1986. BP network generally consists of the input layer, the hidden layer, the output layer, including the two parts of the forward and backward propagation, as shown in figure 1.

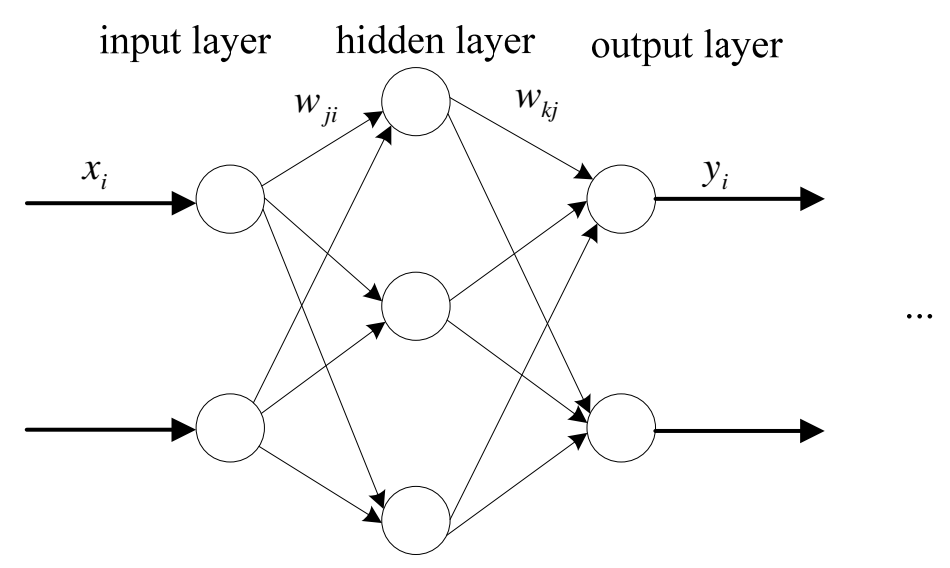

Fig.1 Sketch map of BP neural network with 3 layers

The objective function can reach the minimum by adjusting the weight and threshold with BP neural network method, mainly including two parts of the forward propagation and error reverse correction. The neuron output value $o_{i}$ of input layer is equal to the input value $x_{i}$, and the neuron output of the hidden layer or the output layer can be stated as follows.

$$
O_{j}^{l}=f\left(\sum_{i=1} w_{i j}^{l} o_{i}^{l-1}+\theta_{j}^{l}\right)
$$

In the above formula, $f$ is the transform function and $\theta_{j}^{l}$ is the neuron threshold. If the 
desired output value of the jth neuron in the output layer is $T_{j}$, the total output error of the output layer is as follows.

$$
E=\frac{1}{n} \sum_{j=1}^{n}\left[T_{j}-O_{j}\right]^{2}
$$

The minimum value of the objective function can be solved by using the gradient descent method, and the smoothing factor $\beta$ is introduced to improve the convergence rate. The adjustment formula of network weight and node threshold is as follows.

$$
w_{i j}^{l}(k+1)=w_{i j}^{l}(k)+\eta \delta_{j}^{l} O_{i}^{l-1}+\beta\left(w_{i j}^{l}(k)-w_{i j}^{l}(k-1)\right)
$$

In the above formula, $k$ is the iteration number, $\eta$ is the learning rate, $\delta_{j}^{l}$ is the reverse error signal and the output layer and the hidden layer are respectively as follows.

$$
\begin{gathered}
\delta_{j}^{2}=f^{\prime}\left(\sum_{i=1} w_{i j} o_{i}+\theta_{j}\right)\left(\sum_{i=1} w_{i j} o_{i}+\theta_{j}-f\left(\sum_{i=1} w_{i j} o_{i}+\theta_{j}\right)\right) \\
\delta_{j}^{1}=f^{\prime}\left(\sum_{i=1} w_{i j} o_{i}+\theta_{j}\right) \sum_{n} \delta_{j}^{2} w_{i j}^{2}
\end{gathered}
$$

2.2 Improvement of the traditional BP neural network

The traditional BP neural network often directly uses the method of random weight and threshold, and in order to improve the condition, the genetic algorithm is introduced to optimize the initial weight and the threshold value.

\section{Fault diagnosis theory of BP neural network based on genetic algorithm optimization}

\subsection{Diagnosis principle}

In this paper, the principle diagram of fault diagnosis is shown in Figure 2, and it mainly contains three parts: data acquisition module, fault diagnosis module, fault identification module.

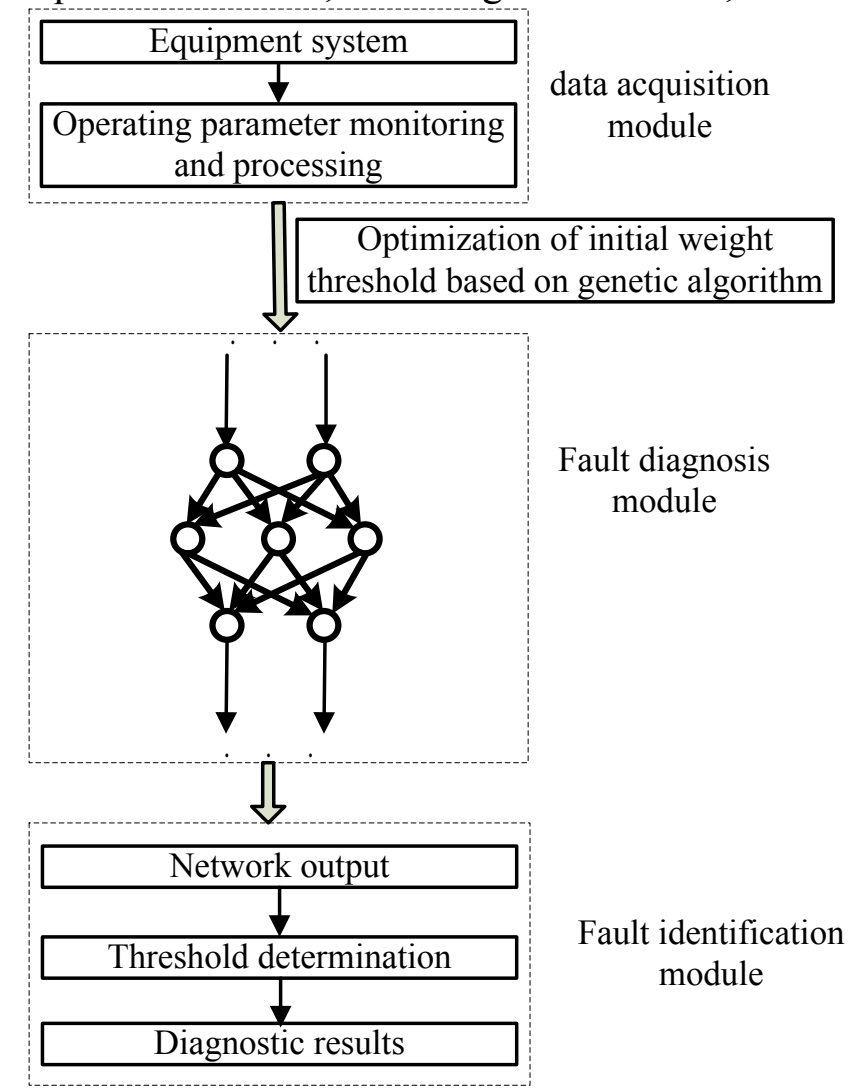

Fig.2 Principle diagram of fault diagnosis

3.2 Establishment of BP neural network diagnosis model

(1)The establishment of the network: in order to establish the neural network, the number of node is firstly determined. In the model of this paper, the input vector is 13 , the output vector is 4 , 
and the input layer node is 13 , the output layer node is 4 . The empirical formula is as follows.

$$
m=2 \times n+1
$$

Among the above formula, $m$ is the number of nodes in the hidden layer, $n$ is the number of nodes in the input layer, and the number of hidden layer nodes is set to 27 . The specific network structure is shown in figure 3.

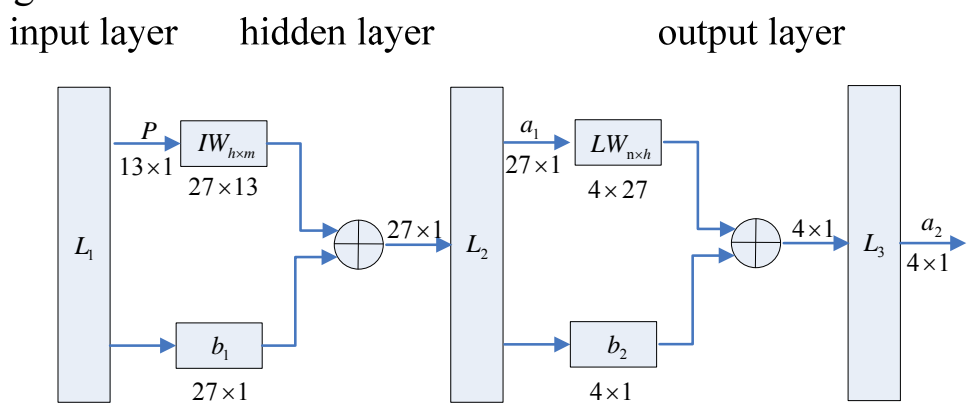

Fig.3 Structure diagram of neural network

notes: $P$-Number of input samples; $L_{1}$-Number of network input layer; $L_{2}$ - Number of neural network hidden layer; $L_{3}-$ Number of neural network output layer; $m-$ Number of input layer neuron; $h$-Number of hidden layer neuron; $n$-Number of output layer neuron; $I W_{h \times m}$ - Weight matrix of the input layer to the hidden layer; $L W_{n \times h}$-Weight matrix of the hidden layer to the output layer; $b_{1} b_{2}$-threshold

(2)Network training: the network is trained after the establishment. In order to speed up the convergence of the network and maintain a high precision, it is needed to set up the appropriate network parameters. For the problem of fault diagnosis, the relevant parameters are determined as follows: the learning rate is 0.01 , the training target is 0.001 , and the training number is 1000 .

3.3 genetic algorithm optimization

The initial weights and thresholds of BP neural network are used as the optimization variables, the output error of the network is used as the fitness of genetic algorithm, and the error is gradually reduced through continuous evolution. When the genetic algorithm runs termination, a set of optimal weights and thresholds are obtained, and the weights and thresholds are transmitted to the BP neural network. By doing so, the fault diagnosis can be carried out. The specific algorithm of this module is shown in figure 4.

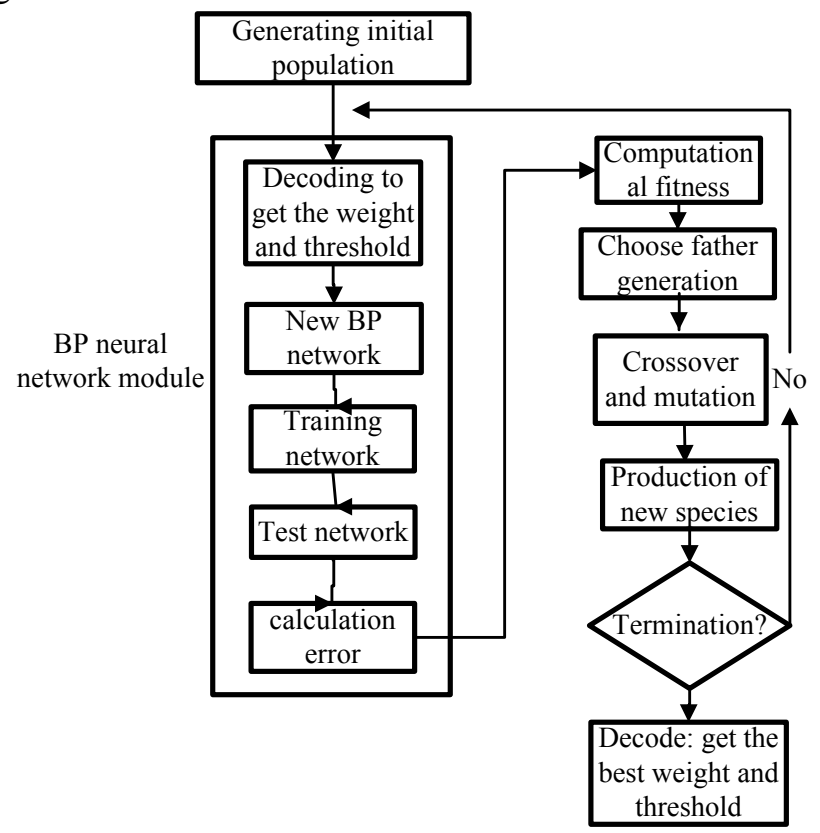

Fig.4 Flow diagram of optimization with genetic algorithm 


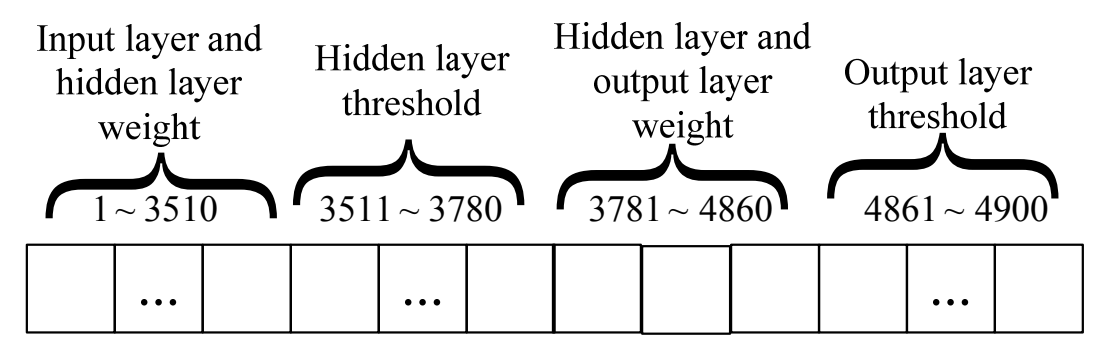

Fig.5 Coding method of chromosome

\section{Simulation calculation}

\subsection{Genetic algorithm optimization results}

The evolution curve of network error is shown in figure 6. From the graph, it can be concluded that the genetic algorithm has a clear evolution before the 50 generation and the 50 generation algorithm begins to converge, and the network error is reduced from the original 0.11 to 0.01 . By doing so, the optimization is achieved. In order to further verify the optimization effect of genetic algorithm, the comparation of the network training error before and after the optimization of genetic algorithm is carried out, and the training error curves are respectively shown in figure 7 and figure 8.

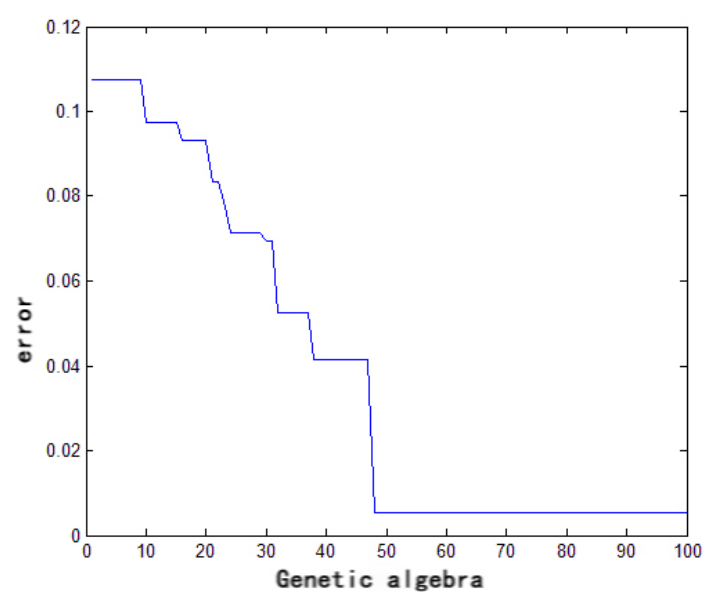

Fig.6 Evolutionary curve of network error

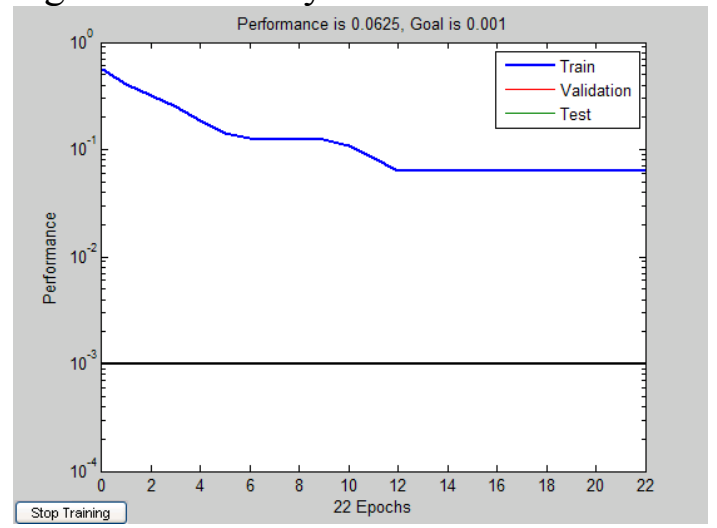

Fig.7 Standard BP network training error curve 


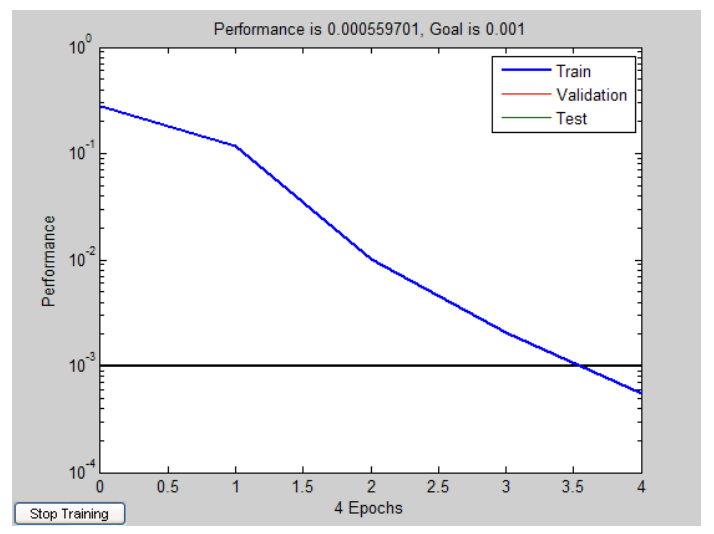

4.2 Fault diagnosis results

Fig.8 Improved BP network training error curve

After the genetic algorithm optimization and network training, the fault diagnosis of equipment can be carried out through the BP network. Four groups of running state are selected and the operation parameter data is entered into the network, and the output results can be obtained through nonlinear mapping, as shown in table 1.

Table1 Diagnosis result of BP network

\begin{tabular}{|c|c|c|c|c|c|}
\hline State & \multicolumn{4}{|c|}{$\begin{array}{l}\text { BP network output result after } \\
\text { optimization }\end{array}$} & $\begin{array}{c}\text { Expected } \\
\text { output }\end{array}$ \\
\hline F1 & 0.9676 & 0.1071 & 0.0169 & 0.0028 & 1000 \\
\hline F2 & 0.0 & 0.9 & 0.0 & 0.1 & 00 \\
\hline F3 & 0.0092 & 0.0030 & 0.9994 & 0.0 & $\begin{array}{llll}0 & 0 & 1 & 0\end{array}$ \\
\hline F4 & 0.0427 & 0.0113 & 0.0019 & 0.9939 & $\begin{array}{llllll}0 & 0 & 0 & 1\end{array}$ \\
\hline
\end{tabular}

\section{Conclusion}

In this paper, the BP neural network is optimized by introducing the genetic algorithm, the fault diagnosis of the equipment is carried out by using the network after optimization, and it has achieved very good results. In order to further verify the correctness of the model, the training and test of the network can be carried out through a large number of different samples, and in order to obtain the best effect, the network can be optimized by using other intelligent algorithm.

\section{References}

[1]GANESH S. An Evaluation of Back - propagation Neural Networks for the Optimal Design of Structural Systems: Part I. Training Procedures[J]. Computer Methods in Applied Mechanics, 2002,191:2873-2886.

[2] Lin Xiaogong, Jiang Xingwei, Liu Tao, et al. Application of genetic neural in stream generator fault diagnosing[J]. Nuclear Power Engineering, 2005, 26(2):199-208.

[3]Nielsen R H. Theory of the Back Propagation Netural Network[J]. Proc of IJCNN, 1989, Vol,2593-2605.

[4] Chunmei $\mathrm{Xu}$. Mechanical servo system based on fuzzy neural network for complex control [J]. Control Engineering .2010:17 (2):146-148.

[5] Zhijun He. LM629-based motor servo control system design [J]. Mechanical design and manufacture 2009 (2) 40-42. 